\title{
ON THE HULL OF A LINEAR DIFFERENTIAL OPERATOR
}

\author{
MAYER HUMI
}

\begin{abstract}
For a system of linear differential equations with almost periodic coefficients with respect to a group $G$ we generalize previous results by Bochner and Sell. Furthermore we explore the relation between the solutions of the original system and those in its hull.
\end{abstract}

I. Introduction. In 1962 Bochner [1] considered the existence of almost periodic (a.p.) solutions to linear ordinary differential equations with a.p. coefficients. More recently Sell [2] discussed the same problem for linear partial differential equations. However, if we examine these two papers from a group theoretical point of view it is easy to realize that $T_{m}$ (= the group of translations in $R^{m}$ ) plays a distinguished role in them. Thus almost periodicity was defined only with respect to (wrt) translations and the operators

$$
D^{\alpha}=\frac{\partial^{\alpha}}{\partial x_{1}^{i_{1}} \cdots \partial x_{m}^{i_{m}}}, \quad i_{1}+\cdots+i_{m}=\alpha,
$$

are invariant wrt $T_{m}$.

In $\S$ II of this paper we generalize the definitions and results of [1], [2] to any group $G$ of affine transformations on $R^{n}$ [3]. In $\S$ III we investigate the relationship between the solutions of a given a.p. differential operator and those in its hull (see definition §II) while in §IV we investigate the subgroup structure of the hull. Finally in $\S \mathrm{V}$ we give some examples.

II. Almost periodicity in $R^{n}$. In this section we generalize the results of [1], [2].

Definition 1. A function $f: R^{m} \rightarrow R^{n}$ is said to be $C^{k}$-bounded if $f=$ $\left(f_{1} \cdots f_{n}\right)$ together with all its derivatives up to and including order $k$ are bounded and uniformly continuous on $R^{m}$.

Definition 2. Let $G$ be a group of affine transformations acting on $R^{m}$. We say that $f: R^{m} \rightarrow R$ is a.p. wrt $G$ if for any sequence $\left\{g_{k}^{\prime}\right\}, g_{k}^{\prime} \in G$, there exists a subsequence $\left\{g_{k}\right\}$ such that $\lim _{k \rightarrow \infty} f\left(g_{k} t\right)$ exists and is uniform in $R^{m}$.

Definition 3. A system of linear differential equations on $R^{m}$ (ordinary or partial) is a set

$$
\sum L_{i j} u_{j}=f_{i}
$$

Presented to the Society, January 18, 1974 under the title On the hull of a partial differential operator; received by the editors March 4, 1975 and, in revised form, January 19, 1976.

AMS (MOS) subject classifications (1970). Primary 35B15, 43A60.

Key words and phrases. Hull of a differential operator, almost periodic solutions. 
where $L_{i j}$ is an arbitrary linear differential operator on $R^{m}$,

$$
L_{i j}=\sum a_{\alpha i j} D^{\alpha}
$$

(the summation is finite).

In the following we denote such a system by $(L, f)$ or $L u=f$.

Definition 4. A system of linear differential equations $L u=f$ on $R^{m}$ is said to be a.p. with respect to $G$ if $D^{\alpha}$ are invariant differential operators wrt $G$ [3] and $a_{\alpha i j}, f_{i}$ are a.p. wrt $G$.

From this definition it is clear that the operators $D^{\alpha}$ will depend on the group $G$ under consideration. If $G=T_{m}$ then $D^{\alpha}$ are those defined in $\S$ I, but they will be different if we consider other groups.

Definition 5. Let $(L, f)$ be a.p. wrt $G$. The hull $H(L, f, G)$ of $(L, f)$ wrt $G$ is the collection of all systems $L^{*} u=f^{*}$ where the functions $a_{\alpha i j}^{*}, f_{i}^{*}$ are related to $a_{\alpha i j}, f_{i}$ by

$$
\begin{aligned}
\lim _{k \rightarrow \infty} a_{\alpha i j}\left(g_{k} t\right) & =a_{\alpha i j}^{*}(t), \\
\lim _{k \rightarrow \infty} f_{i}\left(g_{k} t\right) & =f_{i}^{*}(t),
\end{aligned}
$$

for some sequence $g=\left\{g_{k}\right\}$ which is independent of $\alpha, i, j$ (this limit is pointwise).

REMARKS. 1. In the following we denote the operation that transforms $(L, f)$ into $\left(L^{*}, f^{*}\right)$ by $T(g)$ and write $T(g) L=L^{*}, T(g) f=f^{*}$.

2. In the following almost periodicity will always mean almost periodicity wrt a group $G$ acting on $R^{m}$.

To generalize the results in [1], [2] we start by observing that if $f$ is a.p. then so is $f(g t), g \in G$. Moreover if $\left\{f_{n}(t)\right\}$ is a sequence of a.p. functions which converges uniformly then the limit function is again a.p. From these remarks we obtain the following lemma which is a generalization of Theorem 1 in [1].

LEMmA 1. If $f$ is a.p. wrt $G$ and $\left\{g_{k}^{\prime}\right\},\left\{h_{k}^{\prime}\right\}$ are any infinite sequences in $G$ then there are infinite subsequences,

$$
\left\{g_{l}\right\}=\left\{g_{k_{l}}^{\prime}\right\}, \quad\left\{h_{l}\right\}=\left\{h_{k_{l}}^{\prime}\right\},
$$

for a common sequence of indices $\left\{k_{l}\right\}$ such that

$$
\lim _{s \rightarrow \infty} \lim _{r \rightarrow \infty} f\left(g_{r} h_{s} t\right)=\lim _{k \rightarrow \infty} f\left(g_{k} h_{k} t\right) .
$$

Corollary 1. If $f(t)$ is a.p. then any infinite sequence $\left\{g_{k}^{\prime}\right\}$ contains an infinite subsequence $\left\{g_{k}\right\}$ for which

$$
\lim _{s \rightarrow \infty} \lim _{r \rightarrow \infty} f\left(g_{r} g_{s}^{-1} t\right)=f(t) .
$$

Using the operator $T(g)$ we can rewrite these results in the form

$$
\begin{gathered}
T(g) T(h) f=T(g h) f, \\
T(g) T\left(g^{-1}\right) f=f .
\end{gathered}
$$

Definition 6. We say that $G$ acts effectively on $R^{n}$ if for any $x, y \in R^{n}$ 
there exists $g \in G$ such that $g x=y$ (i.e. $R^{n}$ is a homogeneous space of $G$ ).

From now on all groups $G$ considered will act effectively on $R^{n}$. We now prove the inverse of Lemma 1 (the counterpart of Theorem 1 in [1]).

THEOREM 1. If $f$ is such that any two infinite sequences in $G$ contain infinite subsequences for which the equality

$$
\lim _{s \rightarrow \infty} \lim _{r \rightarrow \infty} f\left(\alpha_{r} \beta_{s} t\right)=\lim _{k \rightarrow \infty} f\left(\alpha_{k} \beta_{k} t\right)
$$

holds and where each of the three limits is assumed to exist for every $t$, then $f(t)$ is a.p.

Proof. If we put $\alpha_{k}^{\prime}=\gamma_{k}^{\prime}, \beta_{k}^{\prime}=e$ (the unit element in $G$ ) then our assumptions imply that every infinite sequence $\left\{\gamma_{k}^{\prime}\right\}$ contains an infinite subsequence $\left\{\gamma_{k}\right\}$ such that $\lim _{k \rightarrow \infty} f\left(\gamma_{k} t\right)=g(t)$ exists for every $t$. We now show that the limit is uniform. If this were not true then nonuniformity would imply that for some $\varepsilon_{0}>0$ there exist monotone sequences of integers $\left\{p_{k}\right\}$, $\left\{q_{k}\right\}$ and a sequence of points $\left\{\delta_{l}\right\}, \delta_{l} \in R^{n}$, such that

$$
\left|f\left(\gamma_{p_{k}} \delta_{k}\right)-f\left(\gamma_{q_{k}} \delta_{k}\right)\right| \geqslant \varepsilon_{0}
$$

However since $G$ is effective on $R^{n}$ there exists $\left\{\beta_{l}\right\}$ such that $\delta_{l}=\beta_{l} \cdot \mathbf{0}$. The assumptions of the theorem imply that the following limits exist (through the choice of subsequences if necessary)

$$
\begin{aligned}
& \lim _{s \rightarrow \infty} \lim _{r \rightarrow \infty} f\left(\gamma_{p_{r}} \beta_{s} \cdot \mathbf{0}\right)=f_{p}(\mathbf{0}), \\
& \lim _{s \rightarrow \infty} \lim _{r \rightarrow \infty} f\left(\gamma_{q_{r}} \beta_{s} \cdot \mathbf{0}\right)=f_{q}(\mathbf{0}),
\end{aligned}
$$

but $\left\{p_{r}\right\},\left\{q_{r}\right\}$ are subsequences of the original sequence, and therefore,

$$
g\left(\delta_{s}\right)=\lim _{r \rightarrow \infty} f\left(\gamma_{p_{r}} \beta_{s} \cdot \mathbf{0}\right)=\lim _{r \rightarrow \infty} f\left(\gamma_{q_{r}} \beta_{s} \cdot \mathbf{0}\right) .
$$

this implies that

$$
f_{p}(\mathbf{0})=f_{q}(\mathbf{0})=\lim _{s \rightarrow \infty} g\left(\delta_{s}\right)
$$

while (2.9) implies

$$
\left|f_{p}(\mathbf{0})-f_{q}(\mathbf{0})\right| \geqslant \varepsilon_{0}>0
$$

which is a contradiction.

Definition 7. A continuous function $f: R^{m} \rightarrow R^{n}$ is said to be almost automorphic (a.a.) if for every sequence $\beta^{\prime}$ there is a subsequence $\beta$ such that the limit $T(\beta) f$ exists and $T\left(\beta^{-1}\right) T(\beta) f=f$.

Using this definition we can define an a.a. system of differential equations and the hull of such a system in exactly the same way that these concepts were introduced for the a.p. case.

With these preparations we can generalize the following results of [1], [2] to any group $G$ which acts effectively on $R^{m}$. The proofs of these results are exactly the same as in [2] except for the generalized meaning of the operators 
$T(g)$ (and the replacement of the + sign by the group multiplication).

Lemma 2 (SelL). Let $f: R^{m} \rightarrow R^{n}$ be a continuous function. Then $f$ is a.p. wrt $G$ if and only if for any two sequences $\beta^{\prime}, \gamma^{\prime}$ there exist subsequences $\beta, \gamma$ such that

(1) $T(\beta) T(\gamma) f$ is a.a. wrt $G$,

(2) $B f=f$,

where $B=T\left((\beta \gamma)^{-1}\right) T(\beta) T(\gamma)$.

Lemma 3 (BochNeR-SELL). Let $L$ be a linear differential operator of order $k$ in $R^{n}$ which is a.a. wrt $G$. If every $C^{k}$-bounded solution of the equation $L^{*} v=0$ for any $L^{*} \in H(L, 0, G)$ is a.a. wrt $G$ then every $C^{k}$-bounded solution of $L^{*} u=f^{*}$. $\left(L^{*}, f^{*}\right) \in H(L, f, G)$ is a.a. wrt $G$.

THEOREM 2. Let L be a linear differential operator of order $k$ which is a.p. wrt $G$. If every $C^{k}$-bounded solution of the equation

$$
L^{*} v=0 \text { for any } L^{*} \in H(L, 0, G)
$$

is a.p. wrt $G$ then every $C^{k}$-bounded solution of

$$
L^{*} u=f^{*}, \quad\left(L^{*}, f^{*}\right) \in H(L, f, G),
$$

is a.p. wrt $G$.

III. The structure of the hull. In this section the phrases a.p. and a.a. will always mean a.p. and a.a. wrt $G$ where $G$ acts effectively on $R^{n}$. Similarly ( $L$, $f$ ) will denote a system of linear differential equations which is a.p. wrt $G$.

LEMma 4. If $(L, f)$ has a $C^{k}$-bounded a.p. solution then every $\left(L^{*}, f^{*}\right) \in$ $H(L, f, G)$ has a $C^{k}$-bounded a.p. solution.

Proof. By definition there exists $T(\gamma)$ such that

$$
T(\gamma) L=L^{*}, \quad T(\gamma) f=f^{*} .
$$

Let $u$ be an a.p. $C^{k}$-bounded solution of $(L, f)$; then

$$
T(\gamma)(L u)=T(\gamma) f
$$

and by taking a subsequence if necessary [1] we obtain that

$$
L^{*}(T(\gamma) u)=f^{*} \text {. }
$$

Thus $T(\gamma) u$ is a solution of $\left(L^{*}, f^{*}\right)$. Moreover $T(\gamma) u$ is an a.p. $C^{k}$-bounded solution as a limit of a $C^{k}$-bounded a.p. function.

LEMMA 5. If all $C^{k}$-bounded solutions of $(L, f)$ are a.p. then any a.a. solution $u^{*}$ of $\left(L^{*}, f^{*}\right)$ is a.p. where $\left(L^{*}, f^{*}\right) \in H(L, f, G)$.

Proof. By definition there exists $T(\gamma)$ such that (3.1) is satisfied. However, $\left(L^{*}, f^{*}\right)$ is a.p. and therefore $T\left(\gamma^{-1}\right) L^{*}=L, T\left(\gamma^{-1}\right) f^{*}=f$. Therefore

$$
T\left(\gamma^{-1}\right)\left(L^{*} u^{*}\right)=L\left(T\left(\gamma^{-1}\right) u^{*}\right)
$$

from which we infer that $T\left(\gamma^{-1}\right) u^{*}$ is a solution of $(L, f)$ and hence a.p. But $u^{*}$ is a.a. and therefore 


$$
u^{*}=T(\gamma) T\left(\gamma^{-1}\right) u^{*}=T(\gamma)\left(T\left(\gamma^{-1}\right) u^{*}\right)
$$

which proves our statement.

Definition 8. By $P(L, f)$ we denote the space of all $C^{k}$-bounded a.p. solutions of $(L, f)$.

THEOREM 3. If $\operatorname{dim} P(L, f)=r$ (finite or infinite) then $\operatorname{dim} P\left(L^{*}, f^{*}\right)=r$ for all $\left(L^{*}, f^{*}\right) \in H(L, f, G)$.

Proof. If $r<\infty$ let $\left\{u_{i}\right\}_{1}^{r}$ be a basis for $P(L, f)$ and let

$$
\left(L^{*}, f^{*}\right) \in H(L, f, G)
$$

then there exist $T(\gamma)$ such that $T(\gamma) L=L^{*}, T(\gamma) f=f^{*}$, and therefore by taking subsequences if necessary,

$$
f^{*}=T(\gamma)\left(L u_{i}\right)=L^{*}\left(T(\gamma) u_{i}\right)
$$

for all $1 \leqslant i \leqslant r$. Thus $T(\gamma) u_{i} \in P\left(L^{*}, f^{*}\right)$. We claim that $\left\{T(\gamma) u_{i}\right\}_{1}^{r}$ are independent. In fact if $\sum c_{i} T(\gamma) u_{i}=0$ then

$$
0=T\left(\gamma^{-1}\right)\left(\sum c_{i} T(\gamma) u_{i}\right)=\sum c_{i} T\left(\gamma^{-1}\right) T(\gamma) u_{i}=\sum c_{i} u_{i}
$$

from which we infer that all $c_{i}=0$. Thus $\operatorname{dim} P\left(L^{*}, f^{*}\right) \geqslant \operatorname{dim} P(L, f)$. But $L \in H\left(L^{*}, f^{*}, G\right)$ and therefore $\operatorname{dim} P(L, f) \geqslant \operatorname{dim} P\left(L^{*}, f^{*}\right)$ and thus $\operatorname{dim}$ $P(L, f)=\operatorname{dim} P\left(L^{*}, f^{*}\right)$.

If $r=\infty$ then the proof above implies that for any $r, \operatorname{dim} P\left(L^{*}, f^{*}\right) \geqslant r$ and thus $\operatorname{dim} P\left(L^{*}, f^{*}\right)=\infty$.

COROLlaRY. If $(L, f)$ is a linear ordinary differential operator of order $k$ whose solutions are $C^{k}$-bounded and a.p., then all solutions of

$$
\left(L^{*}, f^{*}\right) \in H(L, f, G)
$$

are $C^{k}$-bounded and a.p.

IV. Subgroup structure of the hull. Let $A$ be a group of linear transformations acting effectively on $R^{n}$ and $(L, f)$ a system of linear differential equations which is a.p. wrt $A$. In the following we denote by $G, K$ subgroups of $A$ such that $G K=K G$.

Lemma 6. If $u$ is a.p. wrt both $G$ and $K$ then for any sequence $\left\{g_{n}\right\}, g_{n} \in G$, such that $v(t)=\lim _{n \rightarrow \infty} u\left(g_{n} t\right)$ exists, the limit function is a.p. wrt $K$.

Proof. Let $\left\{k_{n}^{\prime}\right\}, k_{n}^{\prime} \in K$, be given. We must show that there exists a subsequence $\left\{k_{n}\right\}$ such that

$$
\lim _{s \rightarrow \infty} v\left(k_{s} t\right)=\lim _{s \rightarrow \infty} \lim _{r \rightarrow \infty} u\left(k_{s} g_{r} t\right)
$$

exists and is uniform in $t$. However this is a direct consequence of the fact that the separate limits are uniform in $t$ by the assumption. 
Corollary. If all $C^{k}$-bounded solutions of $(L, f)$ are a.p. wrt $K$ and $G$ then they are a.p. wrt $B=G K$.

Proof. Let $\left\{b_{n}^{\prime}\right\}, b_{n}^{\prime} \in B$, be given. Since $B=G K, b_{n}^{\prime}=g_{n}^{\prime} k_{n}^{\prime}$. By the previous lemma we infer that there exist subsequences of $\left\{k_{n}^{\prime}\right\},\left\{g_{n}^{\prime}\right\}$ with a common set of indices such that $\lim _{s \rightarrow \infty} \lim _{r \rightarrow \infty} u\left(g_{s} k_{r} t\right)$ exists and is uniform in $t$. It then follows that

$$
\lim _{s \rightarrow \infty} \lim _{r \rightarrow \infty} u\left(g_{s} k_{r} t\right)=\lim _{n \rightarrow \infty} u\left(g_{n} k_{n} t\right)=\lim _{n \rightarrow \infty} u\left(b_{n} t\right)
$$

exists and is uniform in $t$, which proves our statement.

Lemma 7. Let $u^{*}$ be a $C^{k}$-bounded solution of $\left(L^{*}, f^{*}\right) \in H(L, f, K)$ which is a.p. wrt $K$. If all $C^{k}$-bounded solutions of $(L, f)$ are a.p. wrt $G$ and $K$, then $u^{*}$ is a.p. wrt $G$.

Proof. Since $\left(L^{*}, f^{*}\right) \in H(L, f, K)$ there exists $T\left(k^{-1}\right), k=\left\{k_{n}\right\}, k_{n} \in K$, such that $T\left(k^{-1}\right) L^{*}=L, T\left(k^{-1}\right) f^{*}=f$. This implies that

$$
f=T\left(k^{-1}\right)\left(L^{*} u^{*}\right)=L\left(T\left(k^{-1}\right) u^{*}\right)
$$

thus $v=T\left(k^{-1}\right) u^{*}$ is a solution of $(L, f)$ and hence a.p. wrt $G K$. Therefore

$$
T(k) v=T(k) T\left(k^{-1}\right) u^{*}=u^{*}
$$

is also a.p. wrt $G$ by Lemma 6 .

In the following, by a solution of $(L, f)$ we mean a $C^{k}$-bounded solution.

TheOrem 4. Let $\left(L^{*}, f^{*}\right) \in H(L, f, G)$ and $(\bar{L}, \bar{f}) \in H\left(L^{*}, f^{*}, K\right)$. If all solutions of any $(L, f)$ are a.p. wrt $K$, then all solutions of any

$$
(\tilde{L}, \tilde{f}) \in H(L, f, G K)
$$

are a.p. wrt GK.

Proof. Let $(\tilde{L}, \tilde{f}) \in H(L, f, G K)$; then there exists $T(g k)$ such that

$$
T(g k) L=T(g) T(k) L=\tilde{L}, \quad T(g k) f=T(g) T(k) f=\tilde{f} .
$$

If we denote $T(g) L, T(g) f$ by $L^{*}, f^{*}$, respectively, then

$$
\left(L^{*}, f^{*}\right) \in H(L, f, G) \text {. }
$$

By the assumption of the theorem and Lemma 7 we infer that all its $C^{k}$-bounded solutions are a.p. wrt $G$ and $K$. However, $(\tilde{L}, \tilde{f}) \in H\left(L^{*}, f^{*}, K\right)$ and, by the assumptions, all $C^{k}$-bounded solutions of $(\tilde{L}, \tilde{f})$ are a.p. wrt $K$. Lemma 7 now implies that all $C^{k}$-bounded solutions of $(\tilde{L}, \tilde{f})$ are a.p. wrt $G$ and $K$. By the corollary following Lemma 6 this is equivalent to being a.p. wrt $G K$.

As an application of this theorem we consider the following

COROllary. Let $(L, f)$ be a.p. wrt $T_{m}$. If all the solutions of any ( $L^{*}$, $0) \in H\left(L, 0, T_{m}\right)$ are a.p. wrt $T_{m}$ and all the solutions of any

$$
(\bar{L}, 0) \in H\left(L^{*}, 0, T_{l}\right)
$$


are a.p. wrt $T_{l}$ (where $\left.k+l=m\right)$, then all solutions of any operator in $H\left(L, f, T_{m}\right)$ are a.p. wrrt $T_{m}$.

Proof. Direct application of Theorem 4 above and Theorem 1 in [2].

V. Examples. In this section we give some examples of operators to which the theory given above can be applied.

1. Let $G=O(3)$ be the group of rotations in three dimensions; then $G$ is a group of linear transformations acting on $R^{3}$ (not effectively). The Laplace operator $\nabla^{2}=\partial^{2} / \partial x^{2}+\partial^{2} / \partial y^{2}+\partial^{2} / \partial z^{2}$ is an invariant operator wrt $G$. Therefore an operator in which $a_{\alpha i j}, f_{i}$ are a.p. wrt $G$ and $D^{\alpha}=\nabla^{2} \cdots \nabla^{2}$ ( $\alpha$-times) is an a.p. operator wrt $G$.

2. Let $G=E(2)$ be the Euclidean group in two dimensions which consists of rotations and translations in the plane $R^{2}$. $G$ acts effectively on $R^{2}$, and the operator $\nabla^{2}=\partial^{2} / \partial x^{2}+\partial^{2} / \partial y^{2}$ is an invariant operator of $G$.

REMARK. These two examples can be extended naturally to any $R^{n}$. However for higher dimensions the corresponding groups $O(n), E(n)$ have more than one independent invariant operator (these are the Casimir operators of the group) so that $D^{\alpha}$ can then be any monomial in these operators [4].

\section{REFERENCES}

1. S. Bochner, A new approach to almost periodicity, Proc. Nat. Acad. Sci. U.S.A. 48 (1962) p. 2039-2043. MR 26 \#2816.

2. G. R. Sell, $A$ note on almost periodic solutions of linear differential equations, J. Math. Anal. Appl. 42 (1973), 302-312. MR 48 \#463.

3. S. Helgason, Differential geometry and symmetric spaces, Academic Press, New York and London, 1962. MR 26 \#2986.

4. B. G. Wybourne, Classical groups for physicists, Interscience, New York, 1974.

Department of Mathematics, Worcester Polytechnic Institute, Worcester, MASSACHUSETtS 01609 